\title{
ANALYSIS OF LATERAL VIBRATIONS OF THE AXIALLY LOADED HELICAL SPRING
}

\author{
KRZYSZTOF MiCHALCZYK \\ AGH University of Science and Technology, Faculty of Mechanical Engineering and Robotics, Kraków, Poland \\ e-mail: kmichal@agh.edu.pl
}

It is shown in this paper that the proposed concept by Krużelecki and Życzkowski (1990) of the equivalent rod can be applied in calculations of natural lateral vibrations of springs and that the obtained results will be nearer FEM results than the standard model based on the Timoshenko equivalent beam. The model, created on this base, allows one to calculate natural frequencies of the clamped-clamped spring. It is also shown that models based on the equivalent beam concept, which are easier to apply than the models treating the spring as the spatially curved rod, have only a slightly smaller accuracy. It is also indicated that in the most common practice making of manufacturing end coils of springs, the natural frequencies differ significantly from the frequencies calculated by means of all tested methods. The performed simulations show that differences between the first and the second as well as the third and the fourth natural frequency of the spring are small and, therefore, the axially symmetrical equivalent beam model can be used without a large error. The diagram allowing one to determine whether the desired frequencies are lower or higher than the cut-off frequency is developed for the presented model.

Keywords: helical spring vibrations, axially loaded spring,coil spring, Timoshenko equivalent beam

\section{Introduction}

Lateral vibrations of helical springs constitute essential hazards for their operation safety, since they can cause high stress amplitudes and contribute to the loss of stability. On account of that, looking for ways of limiting natural vibrations of helical springs and the development of calculation methods allowing one to determine these limitations efficiency is essential. Equally essential is the accurate determination of natural frequencies.

Problems related to determination the above value can be considered when the spring is treated as a spatially curved rod or using the concept of the equivalent beam. Analyses of spring vibrations treated as the spatially curved rod were presented, among others, by Love (1899), Wittrick (1966), Mottershead (1980), Pearson and Wittrick (1986) Jiang et al. (1991), Yıldırım (1999) Becker et al. (2002), Lee and Thompson (2001), Lee (2007), Stander and Du Preez (1992), Nagaya et al. (1986), Taktak et al. (2008), Yu and Yang (2010). The analyses enabled a relatively accurate description of the effect, but due to problem complexity it was very difficult to apply and - depending on assumptions - to obtain analytical results in a closed form. For practical reasons much more suitable is the equivalent rod or beam model (Haringx, 1949; Della Pietra and Della Valle, 1982; Kobelev, 2014; Michalczyk, 2014). However, the beam model does not take into account several factors related to dynamic effects in springs. It assumes, among others, that the equivalent beam modelling the spring in the case of lateral vibrations is prismatic and has axially symmetrical parameters characterising stiffness and mass distribution. It can be expected that this assumption in the case of clamped-clamped springs (with fixed ends) - the most often met case in practice - can be the reason of significant errors, since the spring lateral 
stiffness is not the same in all directions and its distribution round the axis depends on the partial coil number. The problem of lateral vibrations of helical springs treated as the equivalent beam was considered in the paper by Kobelev (2014). However, only the simplest case, such as the simply-supported beam, was solved in that study.

A new concept of an equivalent rod, which could be applied to analysis of the helical spring static stability was proposed by Krużelecki and Życzkowski (1990). That idea, in contrast to the model of Timoshenko and Gere (1961), took into account the influence of the lead angle of the helix line on its flexural, shearing and compression rigidities. In addition, that concept allowed the analysis of spring buckling in two planes.

The aim of the hereby paper is the analysis of the possibility of application of the Krużelecki and Życzkowski (1990) concept to the determination of lateral vibrations of axially loaded helical springs. The results obtained on the basis of such formulated model will be compared with the classical model of the Timoshenko equivalent beam, with the numerical simulation results and with the results achieved by other authors. The presented model additionally takes into account the non-rotational way of mounting of the end coils.

\section{Analysis}

For an element of the equivalent beam modelling the axially helical spring loaded by a force $P$, as shown in Fig. 1, the following equations of motion can be written (Haringx, 1949)

$$
\frac{\partial N}{\partial \psi}+Q=0 \quad m_{e} \frac{\partial^{2} y}{\partial t^{2}}=N \frac{\partial \psi}{\partial x}-\frac{\partial Q}{\partial x} \quad m_{e} r_{g}^{2} \frac{\partial^{2} \psi}{\partial t^{2}}=N \varphi+Q+\frac{\partial M}{\partial x}
$$

The angle $\psi$ originates only from bending, while the angle $\varphi$ originates only from the equivalent beam shearing

$$
\psi+\varphi=-\frac{\partial y}{\partial x} \quad \frac{\partial \psi}{\partial x}=\frac{M}{\alpha} \quad \varphi=\frac{Q}{\beta}
$$

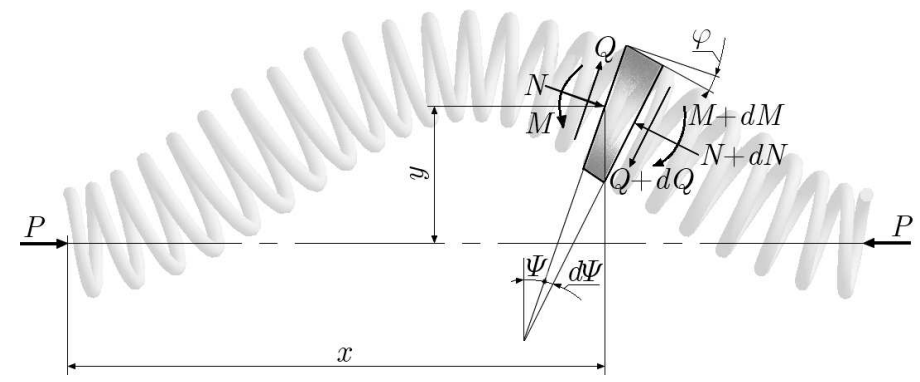

Fig. 1. Model of the equivalent rod for analysis of lateral vibrations of the helical spring

The coefficients $\alpha$ and $\beta$ are up-to-date flexural and shearing rigidities of the equivalent beam, respectively. The spring mass for its length unit is marked by $m_{e}$, while the radius of gyration of the equivalent beam cross-section is marked by $r_{g}$. Limiting the vibration analysis to small deflections only, it can be assumed that $N \approx P$. Making use of $(2.2)$ in $(2.1)$ on this assumption, after transformations, we obtain

$$
\beta\left(1+\frac{P}{\beta}\right) \varphi=m_{e} r_{g}^{2} \frac{\partial^{2} \psi}{\partial t^{2}}-\alpha \frac{\partial^{2} \psi}{\partial x^{2}} \quad-m_{e} \frac{\partial^{2} \varphi}{\partial t^{2}}+\beta \frac{\partial^{2} \varphi}{\partial x^{2}}=m_{e} \frac{\partial^{2} \psi}{\partial t^{2}}+P \frac{\partial^{2} \psi}{\partial x^{2}}
$$


Eliminating $\varphi$ from equations (2.3) and transforming, we obtain the wave equation of lateral vibrations of the spring under the axial static load (Haringx, 1949)

$$
\frac{\partial^{4} \psi}{\partial x^{4}}-\left(\frac{m_{e}}{\beta}+\frac{m_{e} r_{g}^{2}}{\alpha}\right) \frac{\partial^{4} \psi}{\partial x^{2} \partial t^{2}}+\frac{m_{e}^{2} r_{g}^{2}}{\alpha \beta} \frac{\partial^{4} \psi}{\partial t^{4}}+\frac{P}{\alpha}\left(1+\frac{P}{\beta}\right) \frac{\partial^{2} \psi}{\partial x^{2}}+\frac{m_{e}}{\alpha}\left(1+\frac{P}{\beta}\right) \frac{\partial^{2} \psi}{\partial t^{2}}=0
$$

Since we are considering the model with undamped vibrations, separating variables in equation (2.4), the function $\psi(x, t)$ can be written as the product of the amplitude $\Psi(x)$ and the time function $T(t)=\sin \omega t$. Analogous expressions can be also written for the functions $\varphi$ and $y$

$$
\psi(x, t)=\Psi(x) \sin \omega t \quad \varphi(x, t)=\Phi(x) \sin \omega t \quad y(x, t)=Y(x) \sin \omega t
$$

Substituting proper derivatives $(2.5)_{1}$ into equation (2.4) and performing transformations, we obtain the equation for amplitudes in the form

$$
\Psi^{I V}+\left[\left(\frac{m_{e}}{\beta}+\frac{m_{e} r_{g}^{2}}{\alpha}\right) \omega^{2}+\frac{P}{\alpha}\left(1+\frac{P}{\beta}\right)\right] \Psi^{I I}-\left[\frac{m_{e}}{\alpha}\left(1+\frac{P}{\beta}\right) \omega^{2}-\frac{m_{e}^{2} r_{g}^{2}}{\alpha \beta} \omega^{4}\right] \Psi=0
$$

Denoting for simplification

$$
b=\left(\frac{m_{e}}{\beta}+\frac{m_{e} r_{g}^{2}}{\alpha}\right) \omega^{2}+\frac{P}{\alpha}\left(1+\frac{P}{\beta}\right) \quad c=\frac{m_{e}}{\alpha}\left(1+\frac{P}{\beta}\right) \omega^{2}-\frac{m_{e}^{2} r_{g}^{2}}{\alpha \beta} \omega^{4}
$$

The characteristic equation for (2.6), after substituting $\Psi(x)=C \mathrm{e}^{r x}$, takes the form

$$
r^{4}+b r^{2}-c=0
$$

Substituting $q=r^{2}$, we obtain a quadratic equation $q^{2}+b q-c=0$, whose roots are

$$
q_{1}=\frac{1}{2}\left(-b+\sqrt{b^{2}+4 c}\right) \quad q_{2}=\frac{1}{2}\left(-b-\sqrt{b^{2}+4 c}\right)
$$

The expression, which occurs in square roots in (2.9) can be transformed, using (2.7), into

$$
b^{2}+4 c=\frac{m_{e}^{2}}{\alpha^{2} \beta^{2}}\left(\alpha-r_{g}^{2} \beta\right)^{2} \omega^{4}+2 \frac{m_{e}}{\alpha}\left(1+\frac{P}{\beta}\right)\left(\frac{P}{\beta}+\frac{\operatorname{Pr}_{g}^{2}}{\alpha}+2\right) \omega^{2}+\frac{P^{2}}{\alpha^{2}}\left(1+\frac{P}{\beta}\right)^{2}
$$

This expression, similarly as $b$, is higher than null for an arbitrary frequency $\omega$, which means

$$
\bigwedge_{\omega \geqslant 0} q_{2}<0
$$

Thus, the solution form of equation (2.6) depends on sign of the root $q_{1}$. The cut-off frequency value $\omega_{b}$ at which the solution form of equation (2.8) changes, is calculated by equating the right-hand side of equation $(2.9)_{1}$ to null

$$
\omega_{b}=\sqrt{\frac{\beta}{m_{e} r_{g}^{2}}\left(1+\frac{P}{\beta}\right)}
$$

Thus

$$
\bigwedge_{\omega<\omega_{b}} \Psi(x)=C_{1} \cosh \left(k_{1} x\right)+C_{2} \sinh \left(k_{1} x\right)+C_{3} \cos \left(k_{2} x\right)+C_{4} \sin \left(k_{2} x\right)
$$

where

$$
k_{1}=\sqrt{\frac{1}{2}\left(-b+\sqrt{b^{2}+4 c}\right)} \quad k_{2}=\sqrt{\frac{1}{2}\left(b+\sqrt{b^{2}+4 c}\right)}
$$


and

$$
\bigwedge_{\omega>\omega_{b}} \Psi(x)=C_{5} \cos \left(k_{3} x\right)+C_{6} \sin \left(k_{3} x\right)+C_{7} \cos \left(k_{4} x\right)+C_{8} \sin \left(k_{4} x\right)
$$

where

$$
k_{3}=\sqrt{\frac{1}{2}\left(b-\sqrt{b^{2}+4 c}\right)} \quad k_{4}=k_{2}
$$

The way of determining the cut-off frequency $\omega_{b}$ for short, not loaded beams, was studied by Stephen and Puchegger (2006) and Majkut (2009). From the investigations of the author (which are presented in the further part of this paper) it results that even for relatively short springs, the first natural frequencies of lateral vibrations are lower than the cut-off frequency $\omega_{b}$. Therefore, in further considerations, expression (2.12) is used.

The spring deflection angle resulting only from shearing, on the basis of $(2.3)_{1}$, equals

$$
\varphi=\frac{1}{\beta\left(1+\frac{P}{\beta}\right)}\left(m_{e} r_{g}^{2} \frac{\partial^{2} \psi}{\partial t^{2}}-\alpha \frac{\partial^{2} \psi}{\partial x^{2}}\right)
$$

Making use of $(2.2)_{3},(2.5)_{2,3}$ and (2.16) in equation $(2.1)_{2}$, after transformations, we obtain the equation of amplitudes in the form

$$
Y=-\left[\frac{P\left(1+\frac{P}{\beta}\right)+m_{e} \omega^{2} r_{g}^{2}}{m_{e} \omega^{2}\left(1+\frac{P}{\beta}\right)} \Psi^{I}+\frac{\alpha}{m_{e} \omega^{2}\left(1+\frac{P}{\beta}\right)} \Psi^{I I I}\right]
$$

Using derivatives (2.12) in equation (2.17) and transforming, we finally obtain the equation of displacements in the form

$$
\begin{aligned}
& Y(x)=\left(-A k_{1}-B k_{1}^{3}\right) C_{1} \sinh \left(k_{1} x\right)+\left(-A k_{1}-B k_{1}^{3}\right) C_{2} \cosh \left(k_{1} x\right) \\
& +\left(A k_{2}-B k_{2}^{3}\right) C_{3} \sin \left(k_{2} x\right)+\left(-A k_{2}+B k_{2}^{3}\right) C_{4} \cos \left(k_{2} x\right)
\end{aligned}
$$

where, for notation simplification, the following marks are introduced

$$
A=\frac{P\left(1+\frac{P}{\beta}\right)+m_{e} \omega^{2} r_{g}^{2}}{m_{e} \omega^{2}\left(1+\frac{P}{\beta}\right)} \quad B=\frac{\alpha}{m_{e} \omega^{2}\left(1+\frac{P}{\beta}\right)}
$$

The boundary conditions, being the clamped-clamped spring, correspond properly with the real operation conditions of a majority of springs applied in industry and, therefore, such a model will be considered. These conditions are
1) $y(0, t)=0$
2) $y(L, t)=0$
3) $\psi(0, t)=0$
4) $\psi(L, t)=0$

where $L$ is the real height of the spring.

From conditions $\mathbf{1}, \mathbf{3}$ and $\mathbf{4}$, we obtain the following relationships

$$
\begin{aligned}
C_{2} & =\frac{-\cosh \left(k_{1} L\right)+\cos \left(k_{2} L\right)}{\sinh \left(k_{1} L\right)+\frac{A k_{1}+B k_{1}^{3}}{-A k_{2}+B k_{2}^{3}} \sin \left(k_{2} L\right)} C_{1} \quad C_{3}=-C_{1} \\
C_{4} & =\frac{A k_{1}+B k_{1}^{3}}{-A k_{2}+B k_{2}^{3}} C_{2}
\end{aligned}
$$


On the basis of the second boundary condition (2.20), we obtain the frequency equation

$$
\begin{aligned}
0= & \left(-A k_{1}-B k_{1}^{3}\right) C_{1} \sinh \left(k_{1} L\right)+\left(-A k_{1}-B k_{1}^{3}\right) C_{2} \cosh \left(k_{1} L\right) \\
& +\left(A k_{2}-B k_{2}^{3}\right) C_{3} \sin \left(k_{2} L\right)+\left(-A k_{2}+B k_{2}^{3}\right) C_{4} \cos \left(k_{2} L\right)
\end{aligned}
$$

The models proposed by Krużelecki and Życzkowski (1990) have been used for the determination of the equivalent stiffness of the beam modelling the spring. The equivalent flexural rigidity and shear stiffness are expressed by

$$
\alpha=\frac{2 E J \sin \delta}{2+\nu \cos ^{2} \delta} \quad \beta=\frac{E J \sin \delta}{R^{2}\left(1+\nu \sin ^{2} \delta\right)} \frac{2 \pi n}{\int_{\phi_{0}} \sin ^{2} \phi d \phi}
$$

where EJ is the product of Young's modulus and area moment of inertia of the wire section, $\nu$ is Poisson's ratio. The values $\delta$ and $R$ are the lead angle of the helix and half of the diameter of the nominal spring for an arbitrary force $P$, respectively. The angle $\phi$, occurring in $(2.23)_{2}$, is the angle marked by the lead radius between the transverse force vector and an arbitrary cross-section of the spring wire. Since the transverse force direction may not coincide with the direction determined by the spring axis and the beginning of the helix line, the angle coordinate of the spring beginning $\phi_{0}$ can generally differ from zero.

As compared to the widely applied in the references equivalent stiffness, see e.g. Timoshenko and Gere (1961), Guido et al. (1978), in which the lead angle of the helix $\delta$ is assumed as constant and equal to zero, expressions (2.23) allow one to take into account a non-zero value of the angle $\delta$ dependent on the axial force $P$. The relationships given by Krużelecki and Życzkowski (1990) take also into account the nominal spring radius $R$ change as well as the change of the active coils number $n$, under the influence of the force $P$. The authors assumed that the way of supporting the spring ends enables their free rotation. In such a case, during compression of the spring the number of its coils usually decreases (Michalczyk, 2009). Since the wire length is constant, it results in an additional increase in its nominal radius $R$. Its value as well as the value of the up-to-date helix line pitch angle $\delta$ can be determined by rearrangement of the relationships between the torsion and curvature of the helix wire and the force $P$ given by Haringx (1948)

$$
\frac{P R^{2}(1+\nu) \cos \delta}{E J}=\sin \delta \cos \delta-\frac{R}{R_{0}} \sin \delta_{0} \cos \delta_{0} \quad \frac{P R^{2} \sin \delta}{E J}=\cos ^{2} \delta-\frac{R}{R_{0}} \cos ^{2} \delta_{0}
$$

In order to realise the condition of free rotation of the spring ends, one of them has to be supported on a thrust bearing. Only in such a case equations (2.24) can be used for calculating $R$ and $\delta$.

However, most often, springs are supported in a way making free rotations of the end coils impossible and, therefore, this case will be considered further. The number of active coils $n$ of the spring loaded by the force $P$ is equal - due to the clamped-clamped condition - to the number of active coils $n_{0}$ of the not loaded spring. On this assumption and making use of the geometrical relations, it is possible to determine the helix line pitch angle $\delta$ and the spring nominal radius $R$ under load

$$
\delta=\arcsin \left(\left(1-\frac{P}{\gamma_{0}}\right) \sin \delta_{0}\right) \quad R=R_{0} \frac{\cos \delta}{\cos \delta_{0}}
$$

The equivalent shear stiffness $\gamma_{0}$ occurring in equation $(2.25)_{1}$ can be expressed by the equation (Krużelecki and Życzkowski, 1990)

$$
\gamma_{0}=\frac{E J \sin \delta_{0}}{R_{0}^{2}\left(1+\nu \cos ^{2} \delta_{0}\right)}
$$


Knowing the nominal radius $R$ of the axially loaded clamped-clamped spring, it is possible to calculate its equivalent radius of gyration $r_{g}$. In the case of modelling the radius of gyration of cylindrical helical springs, the equivalent rod constitutes the thin-walled cylinder of the average radius equal to the nominal spring radius $R$ and of the mass equal to the spring mass. On this assumption, making use of $(2.25)_{2}$, the equivalent radius of gyration $r_{g}$ can be written in the form

$$
r_{g}=\frac{\sqrt{2}}{2} R_{0} \frac{\cos \delta}{\cos \delta_{0}}
$$

Introducing $(2.25)_{1},(2.26),(2.27)$ into $(2.11),(2.22),(2.23)$, we can calculate cut-off frequencies and natural frequencies of lateral vibrations.

\section{Simulations, results and discussion}

\subsection{Analysis of natural frequencies of lateral vibrations of not axially loaded springs}

Comparisons of the results obtained on the basis of numerical FEM simulations of models (given in references) in which the spring is considered as a spatially curved rod of the classic model of the Timoshenko equivalent beam (applied up-to-date in all investigations concerning lateral vibrations of the spring modelled as the equivalent rod) with the proposed in this paper model using the concept of the equivalent beam given by Krużelecki and Życzkowski (1990) is presented below. As the first example, the spring of the same parameters as those used in work by Lee and Thompson (2001) is analysed. These parameters are as follows: total spring length $L=332 \mathrm{~mm}$, spring nominal radius $R_{0}=65 \mathrm{~mm}$, wire radius $r=6 \mathrm{~mm}$, density $\rho=7800 \mathrm{~kg} / \mathrm{m}^{3}$, number of active coils $n=6$, Young's modulus $E=209000 \mathrm{MPa}$, Poisson's ratio $\nu=0.28$. The first four forms of lateral vibrations of the clamped-clamped spring not loaded by a longitudinal force obtained by the FEM in the ANSYS software are presented in Fig. 2.
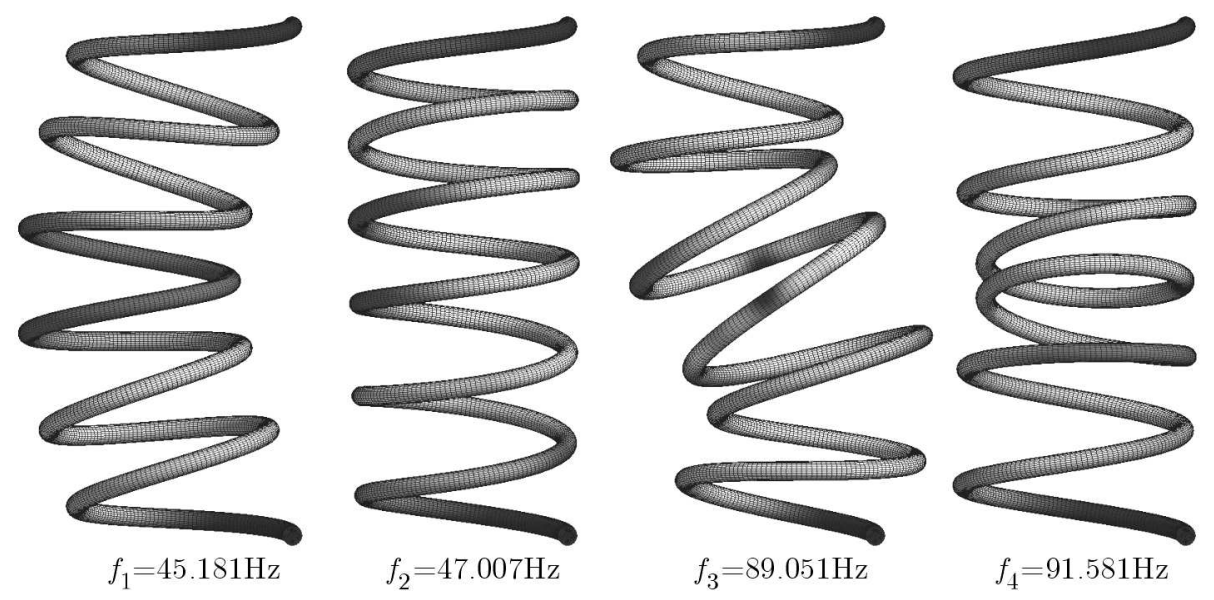

Fig. 2. Forms and frequencies of lateral natural vibrations of the clamped-clamped spring

As can be seen in Fig. 2, the frequency $f_{2}$ is only insignificantly higher than $f_{1}$. In the case of frequencies $f_{4}$ and $f_{3}$, the difference is even smaller.

The comparison of natural frequencies of the spring not loaded by a longitudinal force obtained by means of FEM, the dynamic stiffness method (Lee and Thompson, 2001), transfer matrix method (Pearson, 1982), standard model of the Timoshenko equivalent beam (STB) and the model presented in this paper (MTB) is presented in Table 1. In the methods presented by Lee and Thompson (2001) and Pearson (1982), the spring is treated as a spatially curved rod. The solution, in both methods, is obtained by means of a numerical method. 
It should be noticed, that in methods using the concept of the equivalent rod modelled as acylinder, all frequencies of lateral vibrations are doubled due to the axial symmetry.

Table 1

\begin{tabular}{|l|c|c|c|c|}
\hline & $f_{1}$ & $f_{2}$ & $f_{3}$ & $f_{4}$ \\
\hline \hline FEM model (Fig. 2) & 45.181 & 47.007 & 89.051 & 91.581 \\
\hline Dynamic stiffness method & 45.135 & 46.951 & 88.976 & 91.586 \\
\hline Transfer matrix method & 45.13 & 46.95 & 88.97 & 91.59 \\
\hline Equivalent Timoshenko beam (STB) & 45.975 & 45.975 & 94.088 & 94.088 \\
\hline Model presented in the work (MTB) & 45.764 & 45.764 & 93.62 & 93.62 \\
\hline
\end{tabular}

Comparing the results given in Table 1 it is seen that the application of the modified Timoshenko beam model (MTB) with taking into account non-rotational ends support and using stiffness given in the paper by Krużelecki and Życzkowski (1990) provides, in the case of the analysed not axially loaded spring, results very similar to those ones obtained on the basis of the standard Timoshenko beam model (STB). The largest difference between the frequencies obtained from the FEM simulation and the frequencies obtained from the two last models did not exceed $6 \%$.

The most often occurring case in the engineering practice is the spring of end coils bent and ground off, since only this way of support (except cases of using special spring holders or spring guided on a pin or in a cylindrical sleeve) ensures its stable operation. It can be expected that the way of making the end coils has an influence on the natural frequencies. In order to illustrate this problem, FEM analyses have been performed for a spring of the same parameters as before but with different shapes of neutral coils. The model of such spring and its first four lateral vibration modes are presented in Fig. 3.
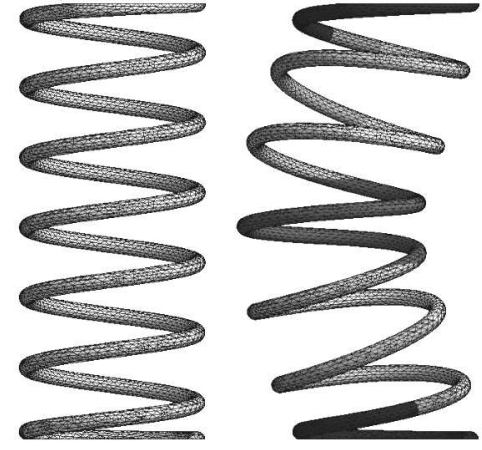

$f_{1}=43.298 \mathrm{~Hz}$

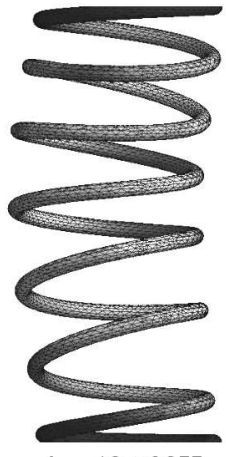

$f_{2}=43.798 \mathrm{~Hz}$

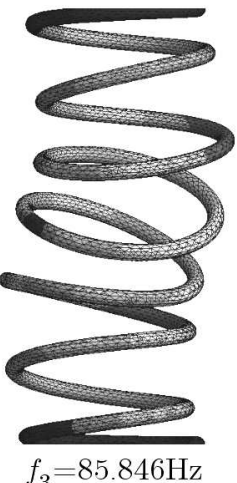

$f_{3}=85.846 \mathrm{~Hz}$

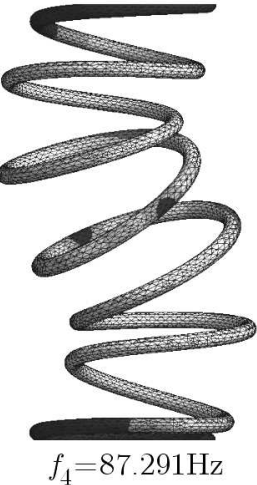

$f_{4}=87.291 \mathrm{~Hz}$

Fig. 3. Forms and frequencies of lateral natural vibrations of the clamped-clamped spring with typical industrial end coils

Comparing data contained in Table 1 and 2, it can be noticed that in the case of the spring with end coils bent and ground off, a better similarity of the FEM results for the models proposed by Lee and Thompson (2001) and Pearson (1982) is not obtained (at least for the first two frequencies) than the results obtained on the basis of the models based on the equivalent beam concept.

Table 2

\begin{tabular}{|c|c|c|c|c|}
\hline & $f_{1}$ & $f_{2}$ & $f_{3}$ & $f_{4}$ \\
\hline \hline FEM model with bent coils (Fig. 3) & 43.298 & 43.798 & 85.846 & 87.291 \\
\hline
\end{tabular}




\subsection{Analysis of natural frequencies of lateral vibrations of axially loaded springs}

Helical springs applied in the industry most often operate under a certain preliminary axial load, usually caused by the supported machine, weight or preliminary tension, e.g. in overload clutches, valves or in variable-speed transmission systems. Thus, the influence of the axial force (approximately statical) on natural frequency vibrations of helical springs is essential. Diagrams of the first four natural frequencies of lateral vibrations obtained (as before) from FEM analyses and from the solution presented in this study are presented in Fig. 4. The FEM simulations were carried out in two stages: in the first one, the statistical analysis was performed at the determined spring load, while in the second, the modal analysis was performed for such a loaded model. Analyses and calculations were performed for the following spring relative deflections: 0; $0.125 ; 0.25 ; 0.375 ; 0.5$. Simulations were carried out in the ANSYS packet, Mechanical APDL module.

As can be seen in Fig. 4, along with an increase in the axial force $P$ and thus an increase in the spring relative deflection, the model presented in this study improves convergence with the FEM in relation to the standard model of the equivalent beam.

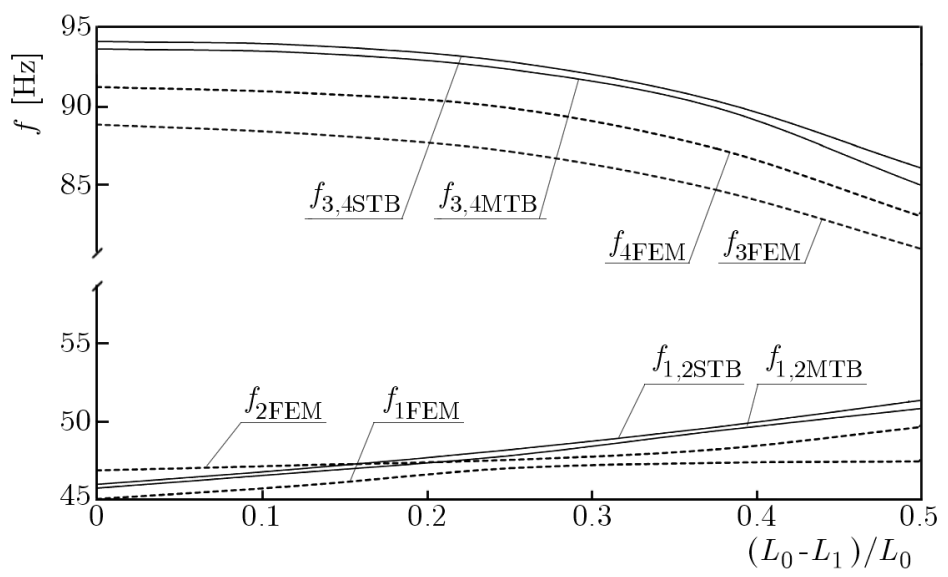

Fig. 4. Comparison of the first four natural frequencies of lateral vibrations obtained from FEM simulations with the first two double frequencies calculated on the basis of the standard model of the equivalent beam (STB) and the model presented in this study (MTB)

\subsection{Cut-off frequencies of lateral vibrations of helical springs according to the presented model}

As has been mentioned above, equations (2.12), (2.18) and (2.22) are applicable only in the case when the cut-off frequency $\omega_{b}$ determined by equation (2.11) is larger than the looked for natural frequencies. Thus, from the point of view of analysis of spring lateral vibrations, it is important to find out whether the looked for vibrations form is above or below the cut-off frequency $\omega_{b}$. This problem was analysed by Guido et al. (1978), where the authors indicated that for slenderness $L_{0} / D_{0}=1$ (or less) natural frequencies always correspond to solution (2.13). However, they did not provide neither slenderness values nor relative deflections at which the successive natural frequencies can be calculated from (2.12). On the basis of the studies performed at various material and geometrical parameters, it can be stated that the spring slenderness and its deflection decide whether the given natural frequency is higher or lower than the cut-off frequency.

The diagram allowing one to determine whether equation (2.12) or (2.14) should be used in calculations of natural frequencies of lateral vibrations is presented in Fig. 5. The up-to-date spring length $L_{1}$ related to the length of a not loaded spring $L_{0}$ is marked in the vertical axis, 
while the not loaded spring slenderness $L_{0} / 2 R_{0}$ in the horizontal axis. If, e.g. we are interested in natural frequencies of lateral vibrations of the clamped-clamped spring of a slenderness being 2.5 and compressed to 0.6 of its initial height (point $A$ in Fig. 5), then the first two double frequencies $\omega_{1,2}$ and $\omega_{3,4}$ can be calculated using the solution in form (2.12) and (2.22), while in order to find the parameters of higher frequencies, the solution in form (2.14) should be used.

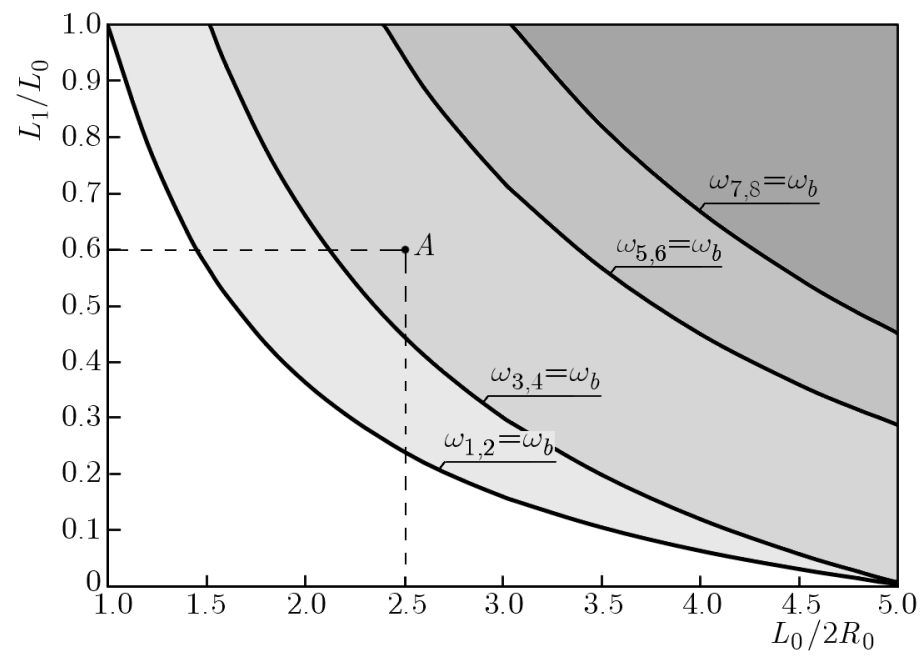

Fig. 5. Curves separating the zones in which the cut-off frequency $\omega_{b}$ is higher than the successive frequencies of lateral natural vibrations

\section{Conclusions}

The modified model of the equivalent beam, presented in this study, allows one to determine form (2.18) and frequencies (2.22) of lateral natural vibrations of the axially loaded spring. The results obtained on the basis of the presented model, using the concept given by Krużelecki and Życzkowski (1990) are slightly closer to the results of the FEM analysis than the standard model based on the Timoshenko equivalent beam. The developed model allows calculation of natural frequencies of the axially loaded spring and supported in a way making free rotations of its end coils impossible. It is also shown that the models based on the equivalent beam concept, easier in applications than models treating the spring as a spatially curved rod, have only insignificantly smaller accuracy. The largest difference between the frequencies obtained from the FEM simulation for the spring without bent end coils (Fig. 2) and the frequencies obtained from the presented model is a little bit above 5\%. Comparing the frequencies obtained from the FEM simulation for the spring without bent end coils (Fig. 2) with the frequencies obtained from the FEM simulation for the spring of typical industrial end coils (Fig. 3) can be noticed that the differences are even larger - the maximum difference for the second frequency exceeded $6 \%$. Thus, the way of making the end coils significantly influences the natural frequencies. In the most often met in practice case of spring end coils being bent and ground off, the natural frequencies differ significantly from the frequencies calculated by all tested methods. The performed simulations indicated also that the differences between the first and the second as well as the third and the fourth natural frequency are small for springs and, therefore, the axially symmetric equivalent beam model can be applied for calculating (without large error) these frequencies. The diagram (Fig. 5) allowing one to determine which form of the solution to equation (2.6) should be applied in calculating frequencies and forms of natural vibrations of the spring of the given slenderness and static deflection has also been developed for the presented model. 


\section{References}

1. Becker L. E., Chassie G. G., Cleghorn W. L., 2002, On the natural frequencies of helical compression springs, International Journal of Mechanical Sciences, 44, 825-841

2. Della Pietra L., Della Valle S., 1982, On the dynamic behavior of axially excited helical springs, Meccanica, 17, 31-43

3. Guido A.R., Della Pietra L., Della Valle S., 1978, Transverse vibrations of helical springs, Meccanica, 13, 2, 90-108

4. HARINGX J.A., 1948, On highly compressible helical springs and rubber rods, and their application for vibration-free mountings, I, Philips Research Reports, 3, 401-449

5. HARIngX J. A., 1949, On highly compressible helical springs and rubber rods, and their application for vibration-free mountings, II, Philips Research Reports, 4, 49-80

6. Jiang W., Jones W.K., Wang T.L., Wu K.H., 1991, Free vibrations of helical springsm, Transactions of ASME, 58, 222-228

7. Kobelev V., 2014, Effect of static axial compression on the natural frequencies of helical springs, $M M M S$, issue 3

8. KruŻEleCKi J., ŻYCZKOWSKi M., 1990, On the concept of an equivalent column in the problem of stability of compressed helical springs, Ingenieur-Archiv, 60, 367-377

9. LEE J., 2007, Free vibration analysis of cylindrical helical springs by the pseudospectral method, Journal of Sound and Vibration, 302, 185-196

10. Lee J., Thompson D. J., 2001, Dynamic stiffness formulation, free vibration and wave motion of helical springs, Journal of Sound and Vibration, 239, 297-320

11. Love A.E.M., 1899, The propagation of waves of elastic displacement along a helical wire, Transactions of the Cambridge Philosophical Society, 18, 364-374

12. Majkut L., 2009, Free and forced vibrations of Timoshenko beams described by single difference equation, Journal of Theoretical and Applied Mechanics, 47, 1,193-210

13. MichalczyK K., 2009, Analysis of helical compression spring support influence on its deformation, The Archive of Mechanical Engineering, 56, 4, 349-362

14. MichalcZYK K., 2014, Influence of the elastomeric coating on parameters of steady state vibrations of coil springs in the resonance and outside it, Journal of Theoretical and Applied Mechanics, $\mathbf{5 2}, 2,507-518$

15. Mottershead J.E., 1980, Finite elements for dynamical analysis of helical rods, International Journal of Mechanical Sciences, 22, 267-283

16. Nagaya K., TAkeda S., NAkATA Y., 1986, Free vibration of coil springs of arbitrary shape, International Journal for Numerical Methods in Engineering, 23, 1081-1099

17. Pearson D., 1982, The transfer matrix method for the vibration of compressed helical springs, Journal of Mechanical Engineering Sciences, 24, 163-171

18. Pearson D., Wittrick W.H., 1986, An exact solution for the vibration of helical springs using a Bernoulli-Euler model, International Journal of Mechanical Sciences, 28, 83-96

19. Stander N., Du Preez R.J., 1992, Vibration analysis of coil springs by means of isoparametric curved beam finie elements, Communications in Applied Numerical Methods, 8, 373-383

20. Stephen N.G., Puchegger S., 2006, On the valid frequency range of Timoshenko beam theory, Journal of Sound and Vibration, 297, 1082-1087

21. Taktak M., Dammak F., Abid S., Haddar M., 2008, A finite element for dynamic analysis of a cylindrical isotropic helical spring, Journal of Materials and Structures, 3, 641-658 
22. Timoshenko S., Gere J.M., 1961, Theory of Elastic Stability, New York and London: McGraw Hill, p. 140

23. Wittrick W.H., 1966, On elastic wave propagation in helical springs. International, Journal of Mechanical Sciences, 8, 25-47

24. Yildrim V., 1999, An efficient numerical method for predicting the natural frequencies of cylindrical helical springs, International Journal of Mechanical Sciences, 41, 919-939

25. Yu A.M., YANG C.J., 2010, Formulation and evaluation of an analytical study for cylindrical helical springs, Acta Mechanica Solida Sinica, 23, 1, 85-94

Manuscript received August 13, 2014; accepted for print March 13, 2015 